\title{
Detection of double orbital angular momentum modes in monochromic dynamic bi-orbital angular momentum multiplexing by ptychography
}

\author{
Zhaohui $\mathrm{Li}^{\mathrm{a}}$ and Delian Liu ${ }^{\mathrm{b}, *}$ \\ ${ }^{a} X i$ 'an University of Science and Technology, School of Communication Engineering, \\ Xi'an, China \\ ${ }^{b}$ Xidian University, School of Physics and Optoelectronic Engineering, Xi' an, China
}

\begin{abstract}
Coherent diffractive ptychographic imaging via a modified multi-state ptychographic algorithm can be used to simultaneously detect double orbital angular momentum (OAM) modes in monochromatic bi-OAM-mode multiplexing. The wavefronts for individual vortex beams modulated by two-dimensional amplitude and temporal pulses and mixed together can be reconstructed independently. In contrast to most other OAM detection methods, this approach solves for the OAM detection in a monochromatic dynamic multi-OAM beam and can be applied as an early step in OAM mode demultiplexing. (C) The Authors. Published by SPIE under a Creative Commons Attribution 4.0 Unported License. Distribution or reproduction of this work in whole or in part requires full attribution of the original publication, including its DOI. [DOI: 10.1117/1.OE.59.11.114104]
\end{abstract}

Keywords: orbital angular momentum; mode multiplexing; ptychography; wavefront reconstruction; multi-state detection.

Paper 20200917 received Jul. 30, 2020; accepted for publication Nov. 12, 2020; published online Nov. 28, 2020.

\section{Introduction}

An optical or electrical vortex beam has a doughnut-shape intensity and a helical phase that carries orbital angular momentum (OAM) per photon. The OAM value for such a beam is equal to $l \hbar$, where $l$ is the OAM mode index and $\hbar$ is the reduced Planck constant. ${ }^{1}$ Because the mode index $l$ can be an unlimited integer value in theory and a beam carrying an OAM mode cannot be created from a linear combination of beams carrying other OAM modes, OAM affords a new physical dimension or a new degree of freedom for data transfer. ${ }^{2-5}$ For example, an enormous information channel capacity can be achieved via OAM encoding, OAM modulation, and OAM division multiplexing. In these applications, OAM beams are generated by techniques that use a spiral phase plate, phase hologram, fiber-based devices, photonic integrated devices, and metamaterials. The beams are loaded with multiple channels of information, mixed and transmitted through a single physical channel. At the receiving end, they are demultiplexed and decoded to restore the information by the same techniques used to generate them but in the opposite way. ${ }^{6-9}$ OAM modes must be known before designing and choosing OAM decoding/demultiplexing. However, in many nonprotocol communication systems, OAM modes are generally unknown. Therefore, it is a promising task to detect the modes of OAM multiplexing in the absence of prior knowledge.

In the past decade, some methods have been proposed to indirectly detect OAM modes based on first measuring the helical phase of the vortex beams and then identifying their topological charge. For phase measuring methods, coherent diffraction imaging (CDI) is a convenient method that does not need interferometry, ${ }^{10,11}$ holographic grating, ${ }^{12,13}$ or other special designed devices, ${ }^{14,15}$ showing great scalability for phase recovering. In CDI, after an object is illuminated by a light beam, the exiting light (either in reflection or transmission) is diffracted for a given distance, producing a diffraction intensity pattern. Then, the complex field of the exiting light can be solved using a computational phase retrieval algorithm with the constraint of diffraction

*Address all correspondence to Delian Liu, dlliu@xidian.edu.cn 
intensity. Consequently, an outstanding algorithm named ptychographic interactive engine has been proposed to overcome the inherent defects of CDI when only one diffraction pattern is recorded; the model is known as coherent diffraction ptychographic imaging (CDPI) ${ }^{16-18}$ In CDPI, a thin-beam scans an object region by region, and two adjacent regions are partially overlapped. The far-field diffraction intensity patterns for the illuminated regions are then recorded. Because the complex solution must be consistent with multiple intensity constraints under overlap illumination, the solution can be rapidly converged to the true solution. In Ref. 19, CDPI was introduced to reconstruct the helical wavefront of a vortex beam using a pinhole (just as for a probe with an aperture boundary and constant intensity) to scan the profile of a vortex beam. A single OAM mode can be recognized from a helical wavefront image. Following the configuration of CDPI, a multi-state ptychographic algorithm (MsPA) was proposed ${ }^{20}$ in which stationary mixed states of any origin, such as a multiwavelength beam ${ }^{21}$ and the dynamics of objects, ${ }^{22}$ can be reconstructed. Consequently, Esashi et al. ${ }^{23}$ used this remarkable method to reconstruct the wavefront of two OAM modes, which exist in a bichromatic vortex beam and are carried on different wavelengths. However, in the iteration process of Ref. 23, the initial guess for the beam should be given according to the prior knowledge for the mode index $l$. However, it is impossible to know the OAM modes in practical applications. Recently, time-resolved imaging ${ }^{24}$ demonstrated that MsPA is suitable not only for spatial incoherent multiple states but also for temporal incoherent multiple states. Wengrowicz et al..$^{25}$ used this method to reconstruct nine frames with nine different helical phase distributions temporally superposed in a beam. However, the nine OAMs still needed to be measured before the experiment to be used as an initial guess for the probe beam in MsPA.

For practical communication applications, the OAMs for a vortex beam can be rapidly varied according to the transmitted signal. Therefore, to design a phase reconstruction approach for a time-varying vortex beam without any prior knowledge of its OAM modes is of great significance. In this paper, CDPI with MsPA is introduced for wavefront reconstruction for a time-varying multi-OAM vortex beam. To remove the requirement for the OAM-modeselective response of the illuminated object, we proposed a modified MsPA for the detection of monochromic double OAM modes. A monochrome bi-OAM beam refers to a single-wave coherent beam that contains two mutually incoherent OAMs. The mutual incoherence means distinct modes will be preserved and no crosstalk occurs between them when they travel mixed. It can be guaranteed by polarization multiplexing, ${ }^{2}$ optical fiber guided mode multiplexing, ${ }^{3,4}$ wavelength division multiplexing, ${ }^{5}$ or time-division multiplexing. ${ }^{25}$ When this beam irradiates an object, the object responds to individual OAM beams uniformly at the same time since a single wavelength is used. The mutual incoherence of OAM beams makes the far-field diffraction intensity of the exited field equal to the sum of the individual intensities obtained from the production of each OAM by the object. Multiple intensity patterns are produced and recorded when the beam scans the object with adjacent illuminated regions overlapping partially. Then, a modified MsPA is performed to reconstruct the wavefront of each OAM mode, with the object function as a known constraint. We found that two OAM modes in a multiplexing beam can be separated independently with high spatial accuracy, despite the modes having a different amplitude modulation or time-varying helical phase. Simulated results show that our proposed algorithm can recover the mode in a time-varying vortex beam, indicating great robustness.

\section{Principle of Multi-OAM Detection by MsPA}

A schematic diagram for detecting multiple OAMs by CDPI is shown in Fig. 1. A monochromatic multi-OAM beam contains multiple optical vortexes with different helical phases in time or space, which are themselves fully coherent but mutually incoherent.

A single typical vortex beam is expressed with polar coordinates $(\rho, \theta)$ as follows:

$$
P_{n}(\rho, \theta)=A_{n} \exp \left(i l_{n} \theta\right),
$$

where $P_{n}$ is the probe function of the $n$ 'th vortex beam and $l_{n}$ is the topological charge of the helical phase or the mode index of the OAM. $A_{n}$ is the amplitude of the beam, which can be a 


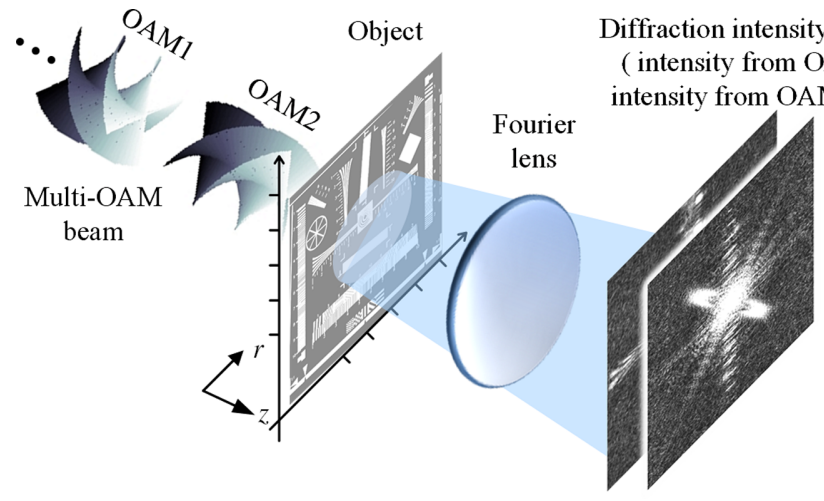

(a)

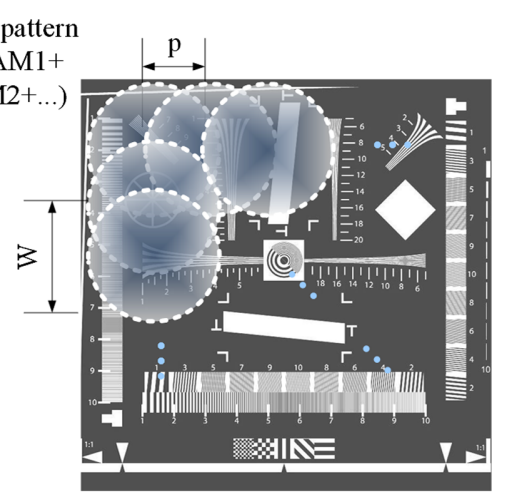

(b)

Fig. 1 Schematic diagram for the detection of multiple OAMs by CDPI: (a) diffraction intensity production from a multi-OAM beam; (b) transverse scanning of an object by the beam.

typical ring-shape-like Laguerre-Gaussian beam or a 2D spatial varying amplitude distribution. The beam is always bounded by an aperture, so there is a support region function when the transmission area of the aperture is $S$.

$$
S_{p}(r)= \begin{cases}1, & r \in S \\ 0, & \text { otherwise }\end{cases}
$$

where $r$ is the position vector transverse to the propagation direction.

The exit wave field generated after an object is illuminated by each vortex beam is written as

$$
\varphi_{m n}(r)=S_{p}(r) \cdot P_{n}(r) \cdot O\left(r-r_{m}\right),
$$

where $O\left(r-r_{m}\right)$ is the object function at the transverse position $r_{m}$. For the purpose of beam measurement, the reference object should have a geometrically simple structure and be free from phase modulation.

A Fourier lens transforms the exit field to the spatial frequency domain at its back focal plane, producing a Fourier spectrum that is proportional to the far-field diffraction of the exit field:

$$
\Psi_{m n}(v)=F\left\{\varphi_{m n}(r)\right\}
$$

where $F$ denotes the Fraunhofer propagation or a Fourier transform operator in a mathematical computer, with the reciprocal coordinate $v$. Therefore, the diffraction intensity distribution obtained at position $r_{m}$ for $N$ mutually incoherent probes is expressed as follows:

$$
I_{m}(v)=\sum_{n=1}^{N} \beta_{n}\left|\Psi_{m n}(v)\right|^{2}=\sum_{n=1}^{N} \beta_{n}\left|F\left\{\varphi_{m n}(r)\right\}\right|^{2}, \quad \beta_{n} \in[0,1], \quad \sum_{n=1}^{N} \beta_{n}=1,
$$

where $\beta_{n}$ denotes the power ratio of the $n$ 'th probe to the total intensity of the beam.

The mixed beam stepwise translates on the object for $M$ times, resulting in $M$ intensity patterns. Since the acquisition time for scanning ptychography is too long for real-time applications, single-shot ptychography has been developed. This uses a microlens array to slit the probe into a beam array, producing ptychographic intensity in a lattice fashion that is obtained in a single camera exposure. ${ }^{18,24,25}$ However, in simulation, we still use the scanning method to obtain the intensities.

The neighboring illuminated regions must overlap partially to ensure convergence for the phase retrieval iteration, as shown in Fig. 1(b). This iteration seeks an exit field constricted by the diffraction intensity patterns, support function boundary, and overlap object modulation. In this iterative phase retrieval algorithm, an operation called "Fourier magnitude projection" is executed, wherein a current exit wave estimate is modified by replacing its Fourier magnitude with the square root of the measured intensity $I_{m}^{\prime}$, while keeping the phase untouched. In MsPA, 
the exit field corresponding to each $P_{n}$ of the Fourier magnitude projection is modified as follows:

$$
\varphi_{m n}^{\prime}(r)=F^{-1}\left\{\Psi_{m n}(v) \cdot \sqrt{I_{m}^{\prime}(v)} / \sqrt{I_{m}(v)}\right\},
$$

where $\varphi_{m n}$ is an estimate of the exit field and $\varphi_{m n}^{\prime}$ is a refreshed estimate after substituting the measured intensity $I_{m}^{\prime}$.

To upgrade and separate $P_{n}$ from $\varphi_{m n}^{\prime}$, the relaxed averaged alternating reflections-algorithm is used to calculate the descent of the gradient for convergence, ${ }^{26,27}$ and the support region constraint in the space domain is applied simultaneously:

$$
P_{n}^{1}=P_{n}+\eta_{p} O^{*}\left(r-r_{m}\right) / \max \left|O\left(r-r_{m}\right)\right|^{2} \cdot\left[S_{p} \cdot\left(2 \varphi_{m n}^{\prime}-\varphi_{m n}\right)-\varphi_{m n}^{\prime}\right],
$$

where the parameter $\eta_{p}$ is an empirical real number with a range of $(0,1)$.

The procedure used for multi-OAM recovery via MsPA is demonstrated in Fig. 2. We list the points that need special attention as follows:

1. There is no special setting for the initialization of $P_{n}^{0}$ at the beginning of the iteration. A random complex matrix is the right choice.

2. The diffraction intensity from each $P_{n}$ should be calculated individually first; only after that can the sum diffraction intensity be obtained. Then, the Fourier projection for each $P_{n}$ can be performed.

3. There is a special ambiguity called mode degeneracy that occurs when two or more modes have equal power; any linear combination of these modes is a possible solution. ${ }^{28}$ To avoid this ambiguity, the power ratios for the probes to be retrieved are estimated to be a set of random fractions, which are different from each other, have a sum of 1 , and are refreshed at every iteration.

4. Every $P_{n}$ is updated with the recorded pattern $I_{m}^{\prime}$ at the $m$ 'th scanned region and set as a new estimate of the probe for the $(m+1)$ 'th position. So before the $(m+1)^{\prime}$ 'th projection of the $k$ 'th iteration, each $P_{n}$ has been updated $M k+m$ times.

5. The iterative cycle is continued until a predefined number of iterations $K$ are completed or an error threshold is achieved. The mean relative error (RE), or the distance between

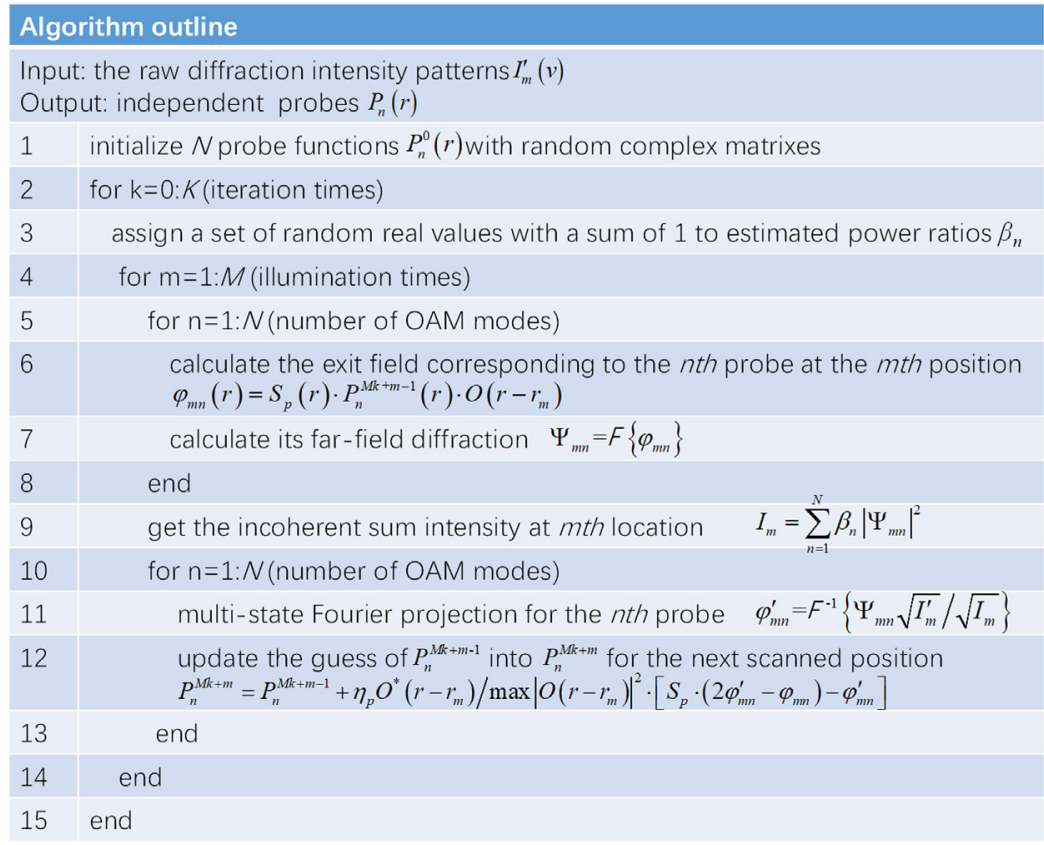

Fig. 2 Algorithm outline for multi-OAM reconstruction. 
the reconstructed diffraction intensity and the actual diffraction intensity at each iteration, is calculated using Eq. (8):

$$
E^{k}=\frac{1}{M} \sum_{m=1}^{M} \frac{\left\|I_{m}^{\prime}-\sum_{n=1}^{N} \beta_{n}\left|\Psi_{m n}\right|^{2}\right\|_{2}}{I_{m}^{\prime}}
$$

\section{Simulated Results}

Some experiments are designed to test the ability of MsPA to detect OAM modes for different types of OAM-based multiplexing. The first experiment tests for the independent reconstruction of multiple stationary OAMs in OAM broad field modulation multiplexing. The second tests the detection of multiple time-varying OAMs in OAM pulse modulation multiplexing. The third experiment performs a synthesis reconstruction for a broad and time-varying multi-OAM beam in an OAM broad field and pulse modulation multiplexing. The scalability of this approach in the case of more than two modes needing to be detected is tested in the fourth experiment. And the performance of the method under non-ideal conditions, involving the imbalance of signal channel, the diffraction intensity patterns polluted with noise, is commented in the final experiments.

\subsection{OAM Broad Field Modulation Multiplexing}

In this case, a helical phase is modulated by a spatially varying amplitude. Such multiple modulated wave fields are incoherently combined together into a beam, propagating synchronously. The wavefront of the beam is stationary at a certain distance. This modulation was employed for OAM holography and image encryption.

The configuration in this simulation experiment is set as Fig. 1. The USAF1951 resolution image with a normalized grayscale of $[0,1]$ shown in Fig. 1(b) is treated as a reference pureamplitude object to be scanned. For the simulation experiment, we generated two complex light probes, with phases that are helixes with different topographical charges and amplitudes that are grayscale images of the digital pattern (same as its topographical charge value). A modulation function $A_{n}$ for the $n^{\prime}$ th probe is a $2 \mathrm{D}$ real value array with a range of $[0,1]$. The mixed beam (two vortex probes are separated schematically) shown in Fig. 3(a) and the complex fields for the wavefronts of the two probes are mapped as grayscale images in Fig. 3(b).

The diameter of the mixed vortex beam $W$ is set to 100 pixels. The scanning pitch $p$ is 25 pixels, which guarantees that the overlap ratio between two neighboring scanned regions is $75 \%$. The size of the reference object $(512 \times 512$ pixels $)$ is much larger than that of the beam, so the probe illumination times, or the amount of diffraction intensity patterns, is $M=16 \times 16$.

First, we generate diffraction patterns by simulating the capture procedure of ptychography. The basis of this operation is an assumption that the OAM modes are mutually incoherent for the OAM multiplexing, which is applied in combination with time-division multiplexing ${ }^{25}$ this proves an absence of interface among OAM modes. At one location, the mixed vortex beam illuminates the object, and the diffraction intensity pattern is the incoherent sum of the far-field diffraction intensity corresponding to each of the OAM probes multiplied by the object, as Eq. (5) described, i.e., calculating the intensities of the two modes and summing them pixel by pixel. The basis of this operation is an assumption that the OAM modes are mutually incoherent for the OAM multiplexing and should be applied in combination with other multiplexing technologies, ${ }^{2-5}$ which proves an absence of interface among OAM modes.

In the simulation and Fourier projection, the size of the diffraction calculation was chosen to be $3 W \times 3 \mathrm{~W}$ to avoid spectrum aliasing caused by the discrete Fourier transform.

Since these light probes carrying OAM modes are assumed to be equal, the real power ratios $\beta_{n}^{\prime}$ are all 0.5 in the generation of raw diffraction patterns $I_{m}^{\prime}$ to ensure that the beam has a normalized power.

Second, we reconstruct the probes via MsPA after 20 iterations. The results obtained are shown in Fig. 3(c), with the initial guess for each probe being of a 2D random complex amplitude field and the power ratios for them being 0.5 . The first row and the third row are the reconstructed complex light fields corresponding to $l=9$ OAM and $l=-9$ OAM, respectively. 


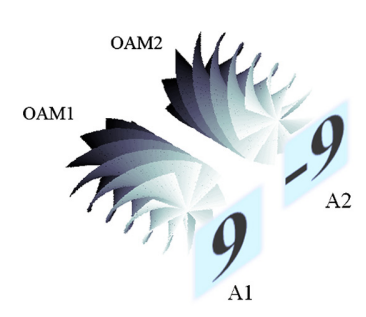

(a)
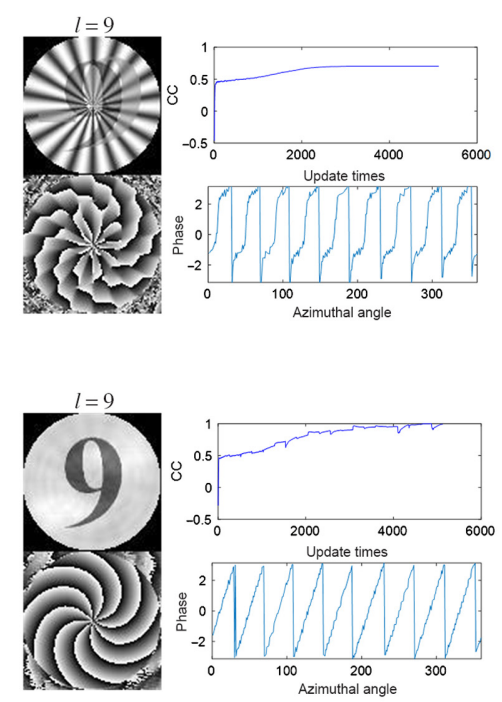

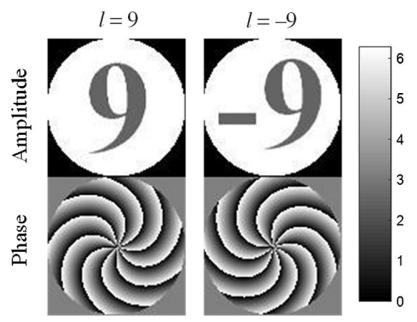

(b)
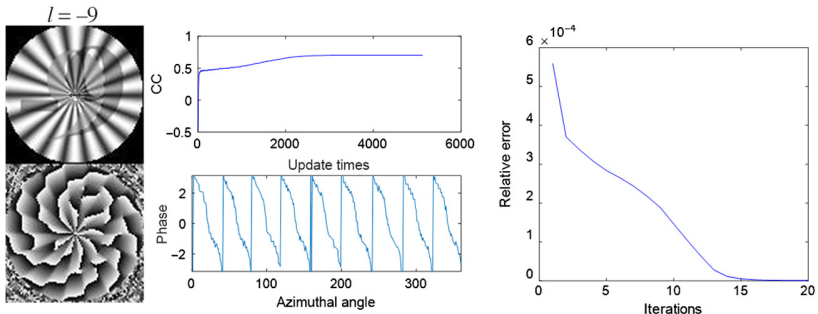

(c)
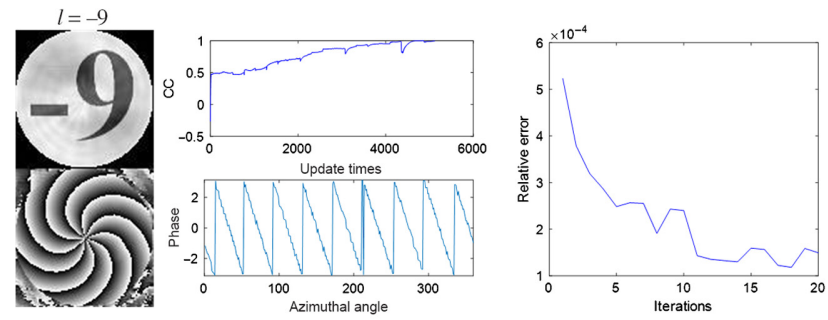

(d)

Fig. 3 Experiment for detecting OAMs in broad field modulation multiplexing: (a) OAM broad field modulation multiplexing; (b) complex fields for the original vortex probes; (c) reconstructed vortex probes when the estimated power ratio of each probe is 0.5 ; and (d) reconstructed vortex probes when the estimated power ratio of each probe is a random fraction refreshed periodically.

A reconstructed amplitude image is followed on the right by a curve of the correlation coefficient (CC) between the original amplitude and the reconstructed amplitude, with the update times (probes are updated at every Fourier projection, $16 \times 16 \times 20=5120$ times). A phase distribution image in the profile is followed on the right by a line of the phase value along the azimuthal angle at a certain radius. To the farthest right is the mean RE of the reconstructed diffraction intensity patterns compared with the actual patterns with iterations.

The reconstructed results obtained by refreshing the power ratios of the probes as random fractions at every iteration cycle are demonstrated in the same format in Fig. 3(d).

One can see from Fig. 3(c) that there is no independent reconstruction of the two OAM modes. The amplitudes of $l=9$ OAM and $l=-9$ OAM are superimposed onto the amplitude of the other OAM and coupled with its phase. That is, a crosstalk between two OAM modes presents in reconstructions. The crosstalk is considered to be caused by a solution ambiguity called mode degeneracy, not from an interface between two modes since they are assumed to be mutually incoherent. This is a typical ambiguity between two modes having equal power. The phase spirals for OAMs are irregular and seriously deformed. The amplitudes in Fig. 3(d) are perfectly recovered with weak background noise.

The CC curves in Fig. 3(d) are higher than that in Fig. 3(c) and are close to 1, which means that the reconstructed amplitudes in Fig. 3(d) are identical to the original amplitudes. The lines for the phase value along the azimuthal angle at a certain polar radius in Fig. 3(d) are straighter and sharper than in Fig. 3(c) and show better agreement with the linear phase item $\exp \left(i l_{n} \theta\right)$. These lines indicate that the reconstructed phases are regular helixes, which is of benefit for topographic charge identity. Despite the RE curve in Fig. 3(d) being greater than that in Fig. 3(c), it still shows an oscillatory convergence to a small value. A comparison of Figs. 3(c) and 3(d) clearly indicates that, for the monochromic beam, MsPA can reconstruct the two OAM modes 
independently and the ambiguity between the two modes is broken by randomizing their estimated power ratios at every iteration.

\subsection{OAM Pulse Modulation Multiplexing}

Another type of OAM division multiplexing that we also take into account is OAM pulse modulation. In this case, a vortex beam is modulated by a string of binary signal pulses, so an OAM is a symbol that is used to represent one bit or more (in an OAM mode coding technique). Multiple information channels are built simultaneously in a physical channel and distinguished by different OAM modes. The modulation function for the $n$ 'th probe at one pulse is expressed as follows:

$$
A_{n}=b_{n}\left(\frac{\rho}{\omega}\right)^{\left|l_{n}\right|} \exp \left(-\frac{\rho^{2}}{\omega^{2}}\right) \exp \left(j \Delta \theta_{n}\right)
$$

where $\omega$ is the waist of the $\mathrm{L}_{-} \mathrm{G}$ beam and $b_{n}$ is a bit value $(1$ or 0$)$ modulating the probe $P_{n}$. The $\exp \left(j \Delta \theta_{n}\right)$ term implies that the pulses start at a time with a random initial azimuthal angle $\Delta \theta_{n}$.

We randomly generated two strings of binary pulses and used them to modulate two different OMA probes, as shown in Fig. 4(a). Figure 4(b) shows the wavefronts for the two probes at one pulse. It is worth noting that the helical phase will rotate to a random azimuthal angle at other pulses. According to time-resolved imaging, an object is illustrated by a burst of pulses that is much faster than the integration time of the sensor, and multiple frames are reconstructed from

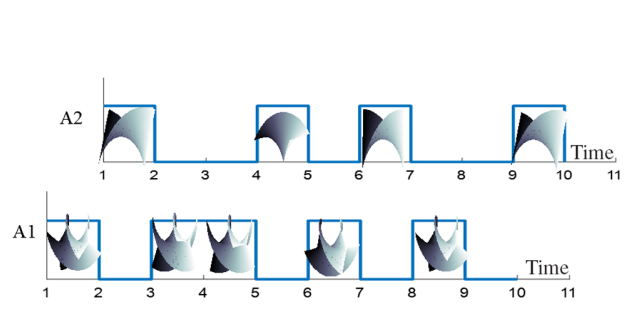

(a)

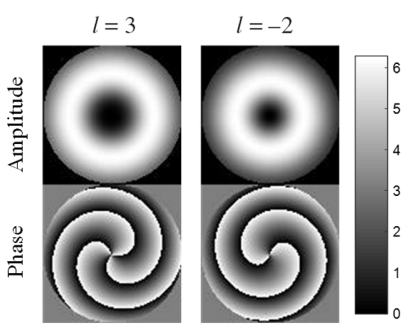

(b)
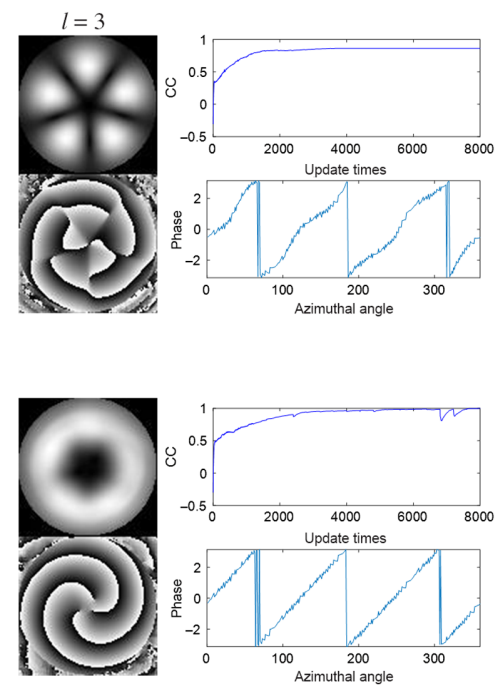
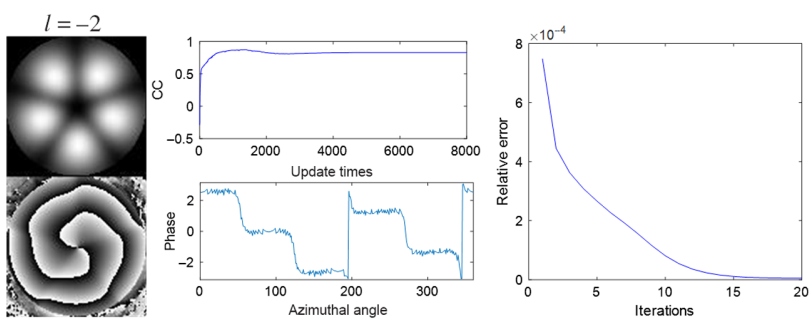

(c)
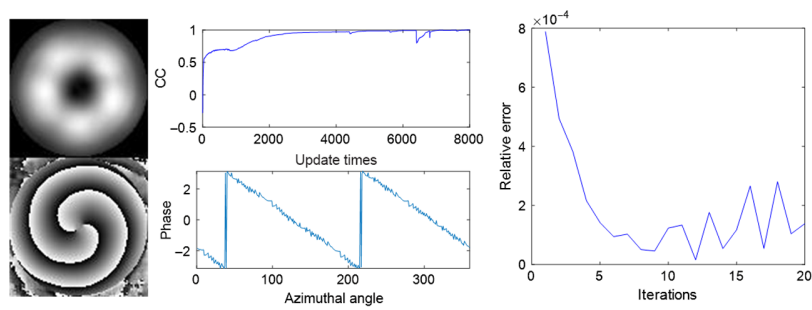

(d)

Fig. 4 Experiment for detecting OAMs in pulse modulation multiplexing: (a) OAM pulse modulation multiplexing; (b) complex fields for OAM probes at one pulse; (c) reconstructed OAM modes when the estimated power ratio of each mode is 0.5 ; and (d) reconstructed OAM modes when the estimated power ratio of each mode is a random fraction refreshed periodically. 
$\mathrm{Li}$ and Liu: Detection of double orbital angular momentum modes in monochromic dynamic...

the intensity patterns obtained from the sum of all of the pulses. ${ }^{24,25}$ The duration of one pulse is assumed to be $1 / 10$ of the exposure time of the sensor used. Therefore, once an object is irradiated by this beam and during sensor exposure for capturing a pattern, the diffraction of an exit field is generated by 10 pulses for each probe, with the pulses having a helical phase with different rotation angles. Therefore, in diffraction patterns generation, diffractions from each pulse for each OMA mode superpose and incoherently add up to a total diffraction intensity pattern, with the real power ratio for all being 0.5 .

The experimental configuration is the same as that used for OAM broad field modulation except for illuminating times of $M=20 \times 20$. It should be noted that the twisting of the phase front into a corkscrew shape results in a doughnut-like intensity profile due to phase singularity at the center of the light beams. As a result, after the object is illuminated by a vortex beam, the center of the exit field is zero, which reduces the redundancy for the intensity data that is necessary in ptychography. Therefore, the amount of intensity patterns should increase.

The reconstruction results obtained for the OAM pulse modulation experiment are shown in Figs. 4(c) and 4(d), with Fig. 4(c) showing the case of all OAM mode power ratios being 0.5 constantly in all iterations, and Fig. 4(d) corresponding to the case of random fractions refreshed at every iteration cycle. Since the amplitudes of the two original OAMs are both bright rings, it is not easy to judge an intermode ambiguity, but one can judge the coupling of amplitude and phase from the petal-like amplitudes in (c). The independent and high-quality reconstruction of the two time-varying OAMs is obtained by setting the power ratios to random fractions, as shown in Fig. 4(d). It is worth noting that this approach can only be used to detect the OAM modes presented in the beam, rather than the timing of their pulses. The fact that a rotating helical phase will not affect OAM mode detection in OAM pulse modulation multiplexing is confirmed in this experiment.

\subsection{OAM Broad Field and Pulse Modulation}

The third OAM multiplexing experiment involves a combination of the two above-mentioned types of multiplexing, OAM broad field and pulse modulation. For this kind of multiplexing, an OAM pulse sequence carries a spatially varying amplitude. That is, each OAM pulse has a rotating helical phase and a specific amplitude, as shown in Figs. 5(a) and 5(b).

Two different OMA probes modulated by different amplitudes in space and by strings of binary pulses in time are combined into a bi-OAM vortex pulsing beam. The experimental configuration used is identical to that in the second experiment, as is the generation of diffraction patterns. The results obtained are demonstrated in Figs. 5(c) and 5(d) in the same style as above.

It should be noted that, in Fig. 5(c), reconstruction under an equal OAM power ratio used in phase retrieval iterations fails since the amplitude images carried by each OAM mode are not clear or independent and the phase distortions are too serious to recognize the helix charge. However, under random OAM power ratios refreshed at each iteration cycle, a perfect reconstruction is obtained, resulting in superior amplitude images and normative helical phases. Double OAM mode detection still works even though the modes have a spatially varying amplitude and time-varying phase.

\subsection{Detection Scalability}

In the above sections, we demonstrated numerical experiments for the detection of two OAM modes in monochromic dynamic bi-OAM multiplexing and the reconstruction of the wavefront of individual vortexes in a mixed beam whenever such modes are amplitude modulated or phase rotated. In addition, it is significant to investigate how many OAM modes this method can detect in parallel. A simulation experiment to detect more than two OAM modes, exactly three here, in OAM multiplexing by our method is performed, with the generation of configuration and diffraction patterns being the same as the ones in broad and pulse modulation multiplexing. Three different OMA probes modulated by different amplitudes in space and by strings of binary pulses in time are combined into a tri-OAM vortex pulsing beam in simulation. Without any prior knowledge of them, the three OAM modes in this beam will be measured and reconstructed by our approach. Figure 6 gives the distribution images of three OAM modes at the original stage in 


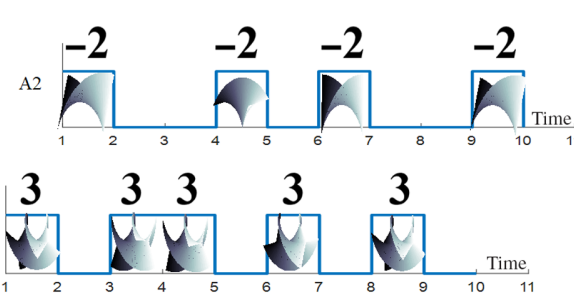

(a)

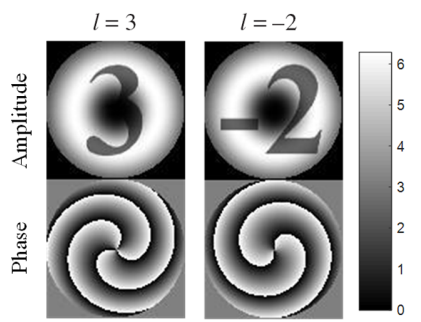

(b)
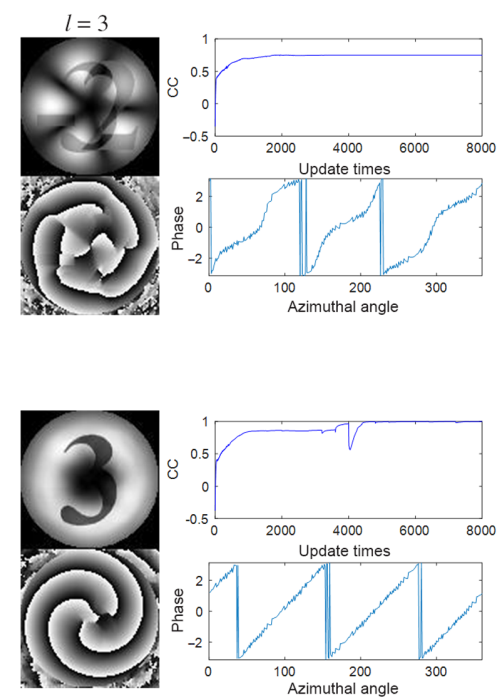
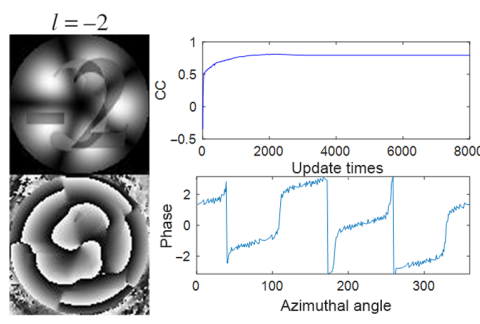

(c)
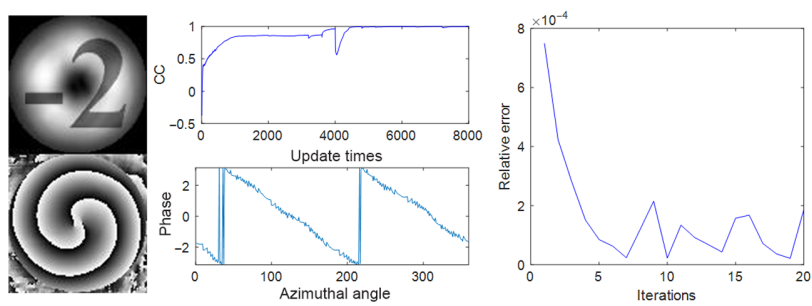

(d)

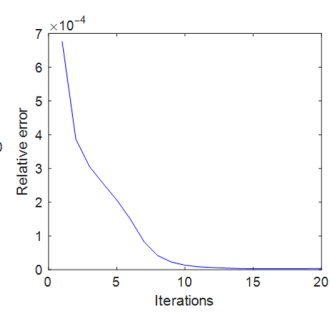

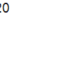




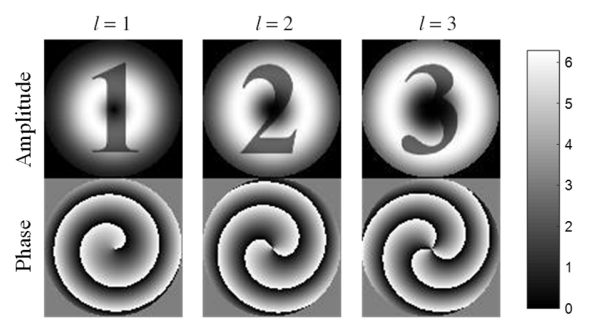

(a)
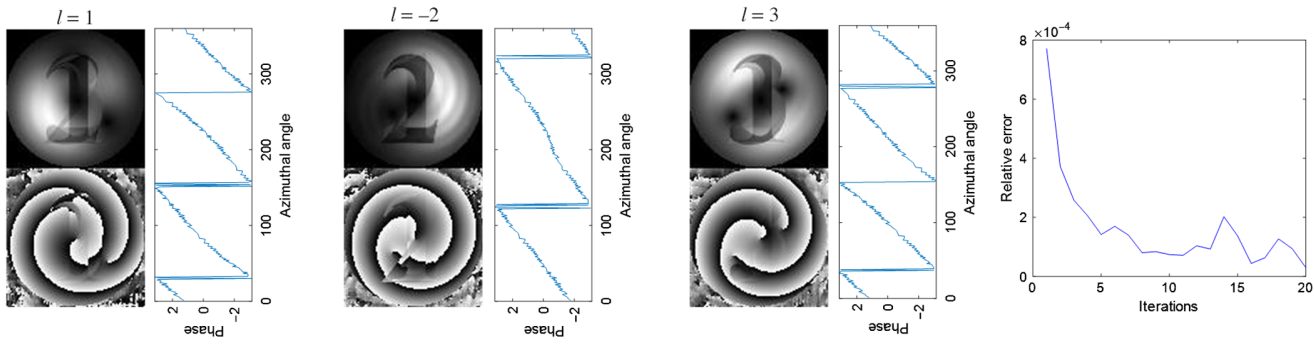

(b)
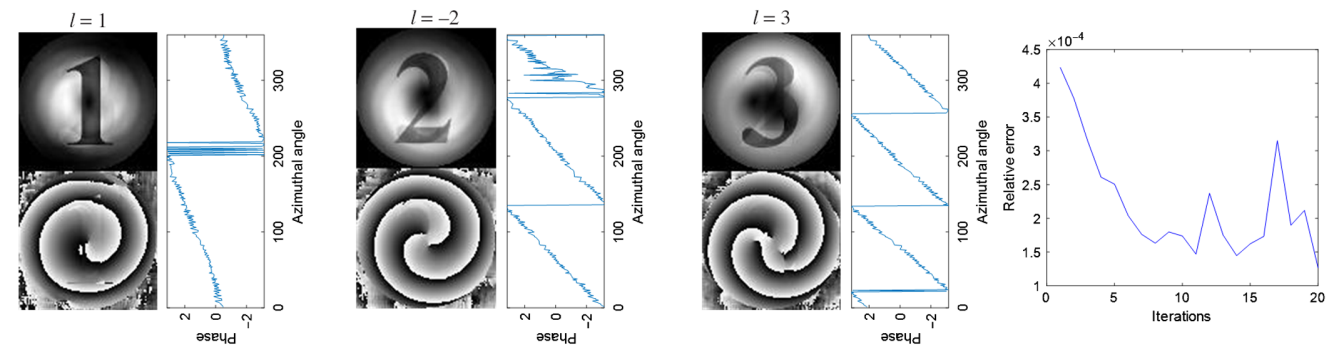

(c)

Fig. 6 Experiment for detecting three OAMs in broad field and pulse modulation multiplexing: (a) complex fields for the three original vortex probes; (b) reconstructed three OAM modes without any prior knowledge of these modes; (c) reconstructed OAM modes when one mode ( $I=1$ probe) is known.

method, the effect of these two main factors on the multi-OAM detection by MsPA will be experimentally examined.

The information channel disequilibrium is discussed first. As mentioned above, the multiplexed signals are always considered to be equal; that is, none of the signals has the dominant power. So the real power ratios for the two OAM probes in previous simulations were assumed to both be 0.5 . An imbalance among the multiplexed signals may occur when carrier OAM beams are emitted from different sources. To simulate this situation, in diffraction intensity patterns generation, the power ratio $\beta_{1}^{\prime}$ for the 1 st OAM probe increases from 0.1 to 0.9 , reverse of the power ratio $\beta_{2}^{\prime}$ for the second OAM probe since the sum of $\beta_{n}^{\prime}$ is 1 . Using these diffraction patterns, the reconstructions by MsPA with random estimated power ratios refreshed periodically are arranged in Fig. 7(a). It is obvious that the lower the power ratio is, the worse the reconstruction quality is. This trend indicates that no reliable reconstruction of an OAM mode can be obtained unless the mode has enough power. Qualitatively, the threshold of the power proportion of a mode in a mixed beam should not be $<0.3$.

In the above experiments, the simulated diffraction patterns are imaged perfectly. But the diffraction patterns are always polluted by noise due to the defects of the imaging system itself. The performance of our method with noisy diffraction intensity patterns will be tested by adding White Gaussian noise to the raw data, or the diffraction intensity patterns plus random intensity matrices. The ratio of the mean power of the raw date to that of the noise is expressed as a signal to noise ratio (SNR). A simulation experiment on bi-OAM detection in broad field and pulse modulation multiplexing, using noisy diffraction intensity patterns with a decreasing SNR, is executed and a part of the results is shown in Fig. 7(b). 


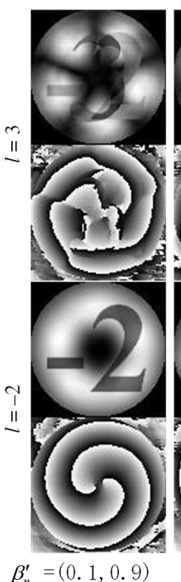

$\beta^{\prime}=(0.1,0.9)$

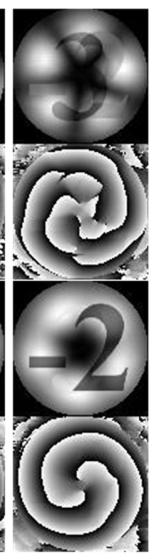

$(0.2,0.8)$

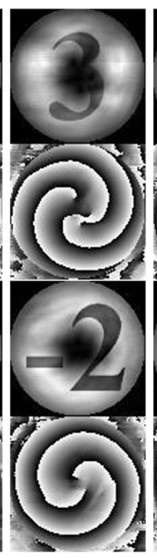

$(0.3,0.7)$

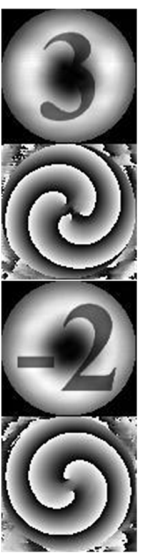

$(0.4,0.6)$

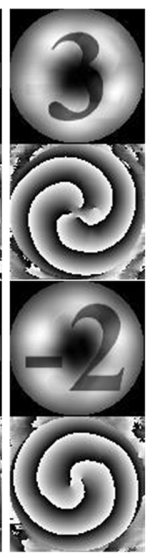

$(0.5,0.5)$

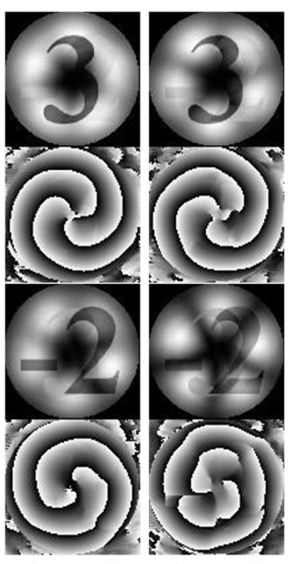

$(0.6,0.4)$

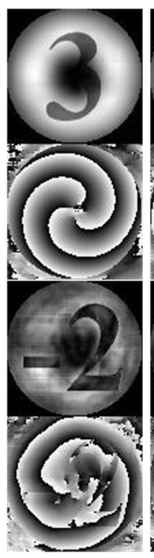

$(0.8,0.2)$

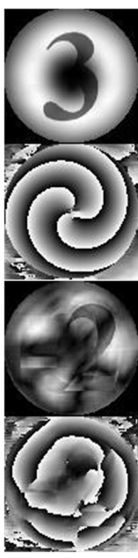

$(0.9,0.1)$

(a)
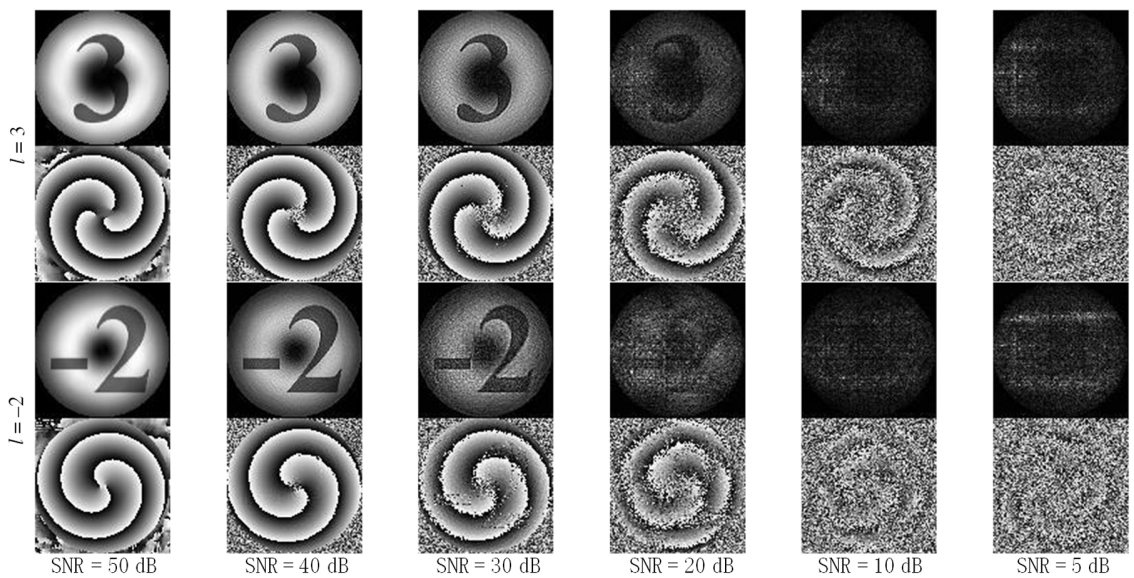

(b)

Fig. 7 Performance evaluation under non-ideal conditions (in broad field and pulse modulation multiplexing): (a) reconstructed OAMs under different real power ratios of probes and (b) reconstructed OAMs with noisy diffraction intensity patterns;

As the SNR decreases, the reconstructed amplitude images carried by each OAM mode become increasingly dimmer and the reconstructed helical phases grow fuzzy, resulting in losing sharp edges where jumping the phase by $2 \pi$. For a reorganization of helical phase and an identification of its topological charge, an SNR in diffraction patterns above $10 \mathrm{~dB}$ should be ensured.

\section{Conclusion and Discussion}

In this work, we investigated the detection of OAM modes in a multiplexing beam. We separated two OAM modes independently, which are of time-varying helical phase and incoherently mixed to propagate simultaneously, by our proposed method. The scalability of the method to detect more than two modes, as well as the robustness on channel imbalance and detector noise, was examined by numerical experiments.

In summary, dynamic multi-OAM mode detection by ptychography affords a new exploitation for OAM multiplexing applications. This approach can be applied as an early step in OAM-mode-demultiplexing to provide a basis for subsequent information channel separation.

\section{Acknowledgments}

The authors thank the National Natural Science Foundation of China (Grant Nos. 61705178, 61774120). The authors declare no conflicts of interest. 


\section{References}

1. A. M. Yao and M. J. Padgett, "Orbital angular momentum: origins, behavior and applications," Adv. Opt. Photonics 3, 161-204 (2011).

2. J. Wang et al., "Terabit free-space data transmission employing orbital angular momentum multiplexing," Nat. Photonics 6(7), 488-496 (2012).

3. N. Bozinovic et al., "Terabit-scale orbital angular momentum mode division multiplexing in fibers," Science 340, 1545-1548 (2013).

4. A. Wang et al., "Directly using 8.8-km conventional multi-mode fiber for 6-mode orbital angular momentum multiplexing transmission," Opt. Express 26(8), 10038-10047 (2018).

5. A. Amphawan and Y. Fazea, "Multidiameter optical ring and Hermite-Gaussian vortices for wavelength division multiplexing-mode division multiplexing," Opt. Eng. 55(10), 106109 (2016).

6. H. Ren et al., "On-chip noninterference angular momentum multiplexing of broadband light," Science 352, 805-809 (2016).

7. Y. Han et al., "Controllable all-fiber generation/conversion of circularly polarized orbital angular momentum beams using long period fiber gratings," Nanophotonics 7, 287-293 (2018).

8. A. Wang, C. Du, and G. Zhu, " $18 \mathrm{~km}$ low-crosstalk OAM + WDM transmission with 224 individual channels enabled by a ring-core fiber with large high-order mode group separation," Opt. Lett. 43(8), 1890 (2018).

9. A. Wang et al., "Demonstration of hybrid orbital angular momentum multiplexing and time-division multiplexing passive optical network," Opt. Express 23(23), 29457-29466 (2015).

10. X. Li et al., "Measuring the fractional topological charge of LG beams by using interference intensity analysis," Opt. Commun. 334, 235-239 (2015).

11. J. Guo et al., "Measuring topological charges of Laguerre-Gaussian vortex beams using two improved Mach-Zehnder interferometers," Opt. Eng. 55(3), 035104 (2016).

12. P. Genevet et al., "Holographic detection of the orbital angular momentum of light with plasmonic photodiodes," Nat. Commun. 3(1), 1278 (2012).

13. H. Ren et al., "Metasurface orbital angular momentum holography," Nat. Commun. 10, 2986 (2019).

14. S. Zheng and J. Wang, "Measuring orbital angular momentum (OAM) states of vortex beams with annular gratings," Sci. Rep. 7, 40781 (2017).

15. V. V. Kotlyar, A. A. Kovalev, and A. P. Porfirev, "Astigmatic transforms of an optical vortex for measurement of its topological charge," Appl. Opt. 56(14), 4095-4104 (2017).

16. H. M. L. Faulkner and J. M. Rodenburg, "Movable aperture lensless transmission microscopy: a novel phase retrieval algorithm," Phys. Rev. Lett. 93, 023903 (2004).

17. A. M. Maidenand and J. M. Rodenburg, "An improved ptychographical phase retrieval algorithm for diffractive imaging," Ultramicroscopy 109, 1256-1262 (2009).

18. X. Pan, C. Liu, and J. Zhu, "Single shot ptychographical iterative engine based on multibeam illumination," Appl. Phys. Lett. 103(17), 171105 (2013).

19. T. Pierre and A. Menzel, "Precise characterization of focused vortex beams," Jpn. J. Appl. Phys. 56(9), 092501 (2017).

20. P. Thibault and A. Menzel, "Reconstructing state mixtures from diffraction measurements," Nature 494(7435), 68-71 (2013).

21. D. J. Batey, D. Claus, and J. M. Rodenburg, "Information multiplexing in ptychography," Ultramicroscopy 138, 13-21 (2014).

22. J. N. Clark et al., "Dynamic imaging using ptychography," Phys. Rev. Lett. 112(11), 113901 (2014).

23. Y. Esashi et al., "Ptychographic amplitude and phase reconstruction of bichromatic vortex beams," Opt. Express 26(26), 34007-34015 (2018).

24. P. Sidorenko, O. Lahav, and O. Cohen, "Ptychographic ultrahigh-speed imaging," Opt. Express 25(10), 10997-11008 (2017).

25. O. Wengrowicz et al., "Experimental time-resolved imaging by multiplexed ptychography," Opt. Express 27(17), 24568-24577 (2019). 
26. S. Marchesini, "Invited article: a [corrected] unified evaluation of iterative projection algorithms for phase retrieval," Rev. Sci. Instrum. 78(1), 011301 (2007).

27. A. Maiden, D. Johnson, and P. Li, "Further improvements to the ptychographical iterative engine," Optica 4(7), 736-745 (2017).

28. P. Li et al., "Breaking ambiguities in mixed state ptychography," Opt. Express 24(8), 9038-9052 (2016).

Zhaohui Li is a lecturer at the Xi' an University of Science and Technology. She received her $\mathrm{PhD}$ in optical engineering from the School of Physics and Optoelectronic Engineering at Xidian University in 2016. Her current research interests include optical communication, optical wave measuring, computational imaging, and optoelectronic systems.

Delian Liu received his BS and MS degrees and his PhD degrees Xidian University, Xi'an, China, in 2003, 2006, and 2008, respectively. He is currently a professor at Xidian University. His research interests include optical imaging, nano-photonics, and remote sensing image analysis. 